\title{
Trends in dementia diagnosis rates in UK ethnic groups: analysis of UK primary care data
}

This article was published in the following Dove Press journal:

Clinical Epidemiology

\author{
Tra My Pham' \\ Irene Petersen ${ }^{1,2}$ \\ Kate Walters' \\ Rosalind Raine ${ }^{3}$ \\ Jill Manthorpe ${ }^{4}$ \\ Naaheed Mukadam ${ }^{5}$ \\ Claudia Cooper ${ }^{5}$
}

'Department of Primary Care and Population Health, University College London, London, UK; ${ }^{2}$ Department of Clinical Epidemiology, Aarhus University Hospital, Aarhus, Denmark; ${ }^{3}$ Department of Applied Health Research, University College London, London, UK; ${ }^{4}$ Social Care Workforce Research Unit, King's College London, London, UK; ${ }^{5}$ Division of Psychiatry, University College London, London, UK
Correspondence: Claudia Cooper Division of Psychiatry, University College London, Maple House - sixth floor, I49 Tottenham Court Road, London WIT 7NF, UK

Email claudia.cooper@ucl.ac.uk
Objectives: We compared incidence of dementia diagnosis by white, black, and Asian ethnic groups and estimated the proportion of UK white and black people developing dementia in 2015 who had a diagnosis for the first time in a UK-wide study.

Methods: We analyzed primary care electronic health records from The Health Improvement Network database between 2007 and 2015 and compared incidence of dementia diagnosis to dementia incidence from community cohort studies. The study sample comprised of 2,511,681 individuals aged 50-105 years who did not have a dementia diagnosis prior to the start of follow-up. Results: A total of 66,083 individuals had a dementia diagnosis $(4.87 / 1,000$ person-years at risk, 95\% CI 4.83-4.90); this incidence increased from 3.75 to 5.65/1,000 person-years at risk between 2007 and 2015. Compared with white women, the incidence of dementia diagnosis was $18 \%$ lower among Asian women (adjusted incidence rate ratio (IRR) 0.82, 95\% CI 0.72-0.95) and 25\% higher among black women (IRR 1.25, 95\% CI 1.07-1.46). For men, incidence of dementia diagnosis was $28 \%$ higher in the black ethnic group (IRR 1.28, 95\% CI 1.08-1.50) and $12 \%$ lower in the Asian ethnic group (IRR 0.88, 95\% CI 0.76-1.01) relative to the white ethnic group. Based on diagnosis incidence in The Health Improvement Network data and projections of incidence from community cohort studies, we estimated that $42 \%$ of black men developing dementia in 2015 were diagnosed compared with $53 \%$ of white men.

Conclusion: People from the black ethnic group had a higher incidence of dementia diagnosis and those from the Asian ethnic group had lower incidence compared with the white ethnic group. We estimated that black men developing dementia were less likely than white men to have a diagnosis of dementia, indicating that the increased risk of dementia diagnosis reported in the black ethnic group might underestimate the higher risk of dementia in this group. It is unclear whether the lower incidence of dementia diagnosis in the Asian ethnic group reflects lower community incidence or underdiagnosis. A cohort study to determine this is needed.

Keywords: dementia, ethnicity, primary care, electronic health records

\section{Introduction}

Around 46.8 million people worldwide have dementia. Based on projected demographic changes, this is expected to increase to 131.5 million by $2050 .{ }^{1}$ The majority of recent population-based studies have reported stable or declining age-adjusted dementia incidence over time. These included a large UK community-based cohort study of people aged $\geq 65$ years, which reported a $20 \%$ drop in age-stratified dementia incidence over 20 years, with an overall incidence of 17.7/1,000 patient-years at risk (PYAR) between 2008 and $2011 .^{2}$ Reflecting the likelihood of people who have dementia receiving 
a diagnosis, a previous study from The Health Improvement Network (THIN) database found a stable incidence of dementia diagnosis in primary care between 1990 and 2007 (3-4/1,000 PYAR). ${ }^{3}$ There is currently no evidence regarding how the likelihood of receiving a diagnosis may differ between people living with dementia from different ethnic groups, although some evidence suggests that people from black and minority ethnic (BME) groups present later in their illness. ${ }^{4}$ This suggests that access to timely diagnosis may be inequitable, preventing some BME people from benefiting from intervention and treatment as early as their white British counterparts.

In 2014, the Alzheimer's Society (England and Wales) concluded that more epidemiological research is urgently required to clarify dementia risk among BME groups. ${ }^{5}$ Incidence of dementia in BME people might differ from the majority population for several reasons. For instance, socially and culturally determined dietary and exercise patterns may affect dementia risk. ${ }^{6}$ Varying immigration histories will involve different exposures to host culture and risks, eg, social integration. Socioeconomic predictors of dementia, such as less formal education, lower income, and worse occupational conditions, are overrepresented in BME groups. Prevalence of genetic variants linked to dementia, such as ApoE- $\varepsilon 4$ genotype, differ across ethnic subgroups, with lower prevalence reported in Asian-American subgroups compared with the majority US population. ${ }^{7}$ Black and Asian ethnic groups experience more cardiometabolic risk factors, such as diabetes and obesity, which are dementia risk factors. ${ }^{8}$ Few community-based cohort studies have evaluated dementia incidence or prevalence in BME groups. In a London (UK) study, dementia prevalence was higher in African-Caribbean compared with older white people after adjusting for age and socioeconomic status (OR 3.1, 95\% CI 1.3-7.3). ${ }^{9}$ In the only epidemiological studies to report dementia incidence in the Asian ethnic group, US-based studies have found that dementia incidence is similar or lower among Japanese-Americans compared with Americans from white ethnic groups. ${ }^{7,10}$

Dementia diagnosis rates may also differ among ethnic groups because of differences in the likelihood of people living with dementia receiving a diagnosis. The best current evidence for ethnic differences in dementia diagnosis come from a large Californian (US) study. In data from patients aged $\geq 60$ years recorded in a large insurance database from 2000 to 2013, which included 3\% of the state population and was broadly representative of the local area, age-standardized dementia incidence was 19.4/1,000 PYAR for white nonLatino ethnic groups and 17.2/1,000 PYAR for the Asian ethnic group, ${ }^{7}$ with the highest dementia incidence reported for African-Americans (26.6/1,000 PYAR) ${ }^{11}$ Among the Asian ethnic group, rates for South Asian people were lower than for other Asian backgrounds (12.1/1,000 PYAR). ${ }^{7}$

In this study, we reported the incidence of dementia diagnosis in UK primary care between 2007 and 2015 and incidence by ethnic group for the first time in a sample broadly representative of the UK population. Using UK census data and current best evidence of community dementia incidence, we estimated the incidence of dementia in people of white and black ethnicities, and based on this, the proportion of white and black people developing dementia who received a diagnosis. We examined the hypothesis that black people developing dementia would be less likely to receive a diagnosis than people from the white ethnic group. There is currently no data available on the incidence of dementia in Asian people living with dementia in the UK.

\section{Aims}

Our aims were to report the incidence of dementia diagnosis over time (2007-2015) in UK primary care; to report the incidence of dementia diagnosis by white, black, and Asian ethnic groups in UK primary care for the first time; and to compare the community incidence of dementia with incidence of dementia diagnosis in 2015 , in order to examine our hypothesis that black people with dementia would be less likely to receive a diagnosis than people from the white ethnic group. ${ }^{5,9}$

\section{Methods}

\section{Data source}

We used THIN database,${ }^{12}$ one of the UK's largest databases of primary care electronic health records, which has been shown to be broadly representative of the UK population. ${ }^{13}$ At the time of data collection, the database contained 645 participating general practices contributing data from $>14$ million patients. THIN holds longitudinal records of patients' medical conditions, symptoms, diagnoses, and prescriptions which are recorded during routine primary care consultations from the time of registration with the practices. The Read code hierarchical system is used to record clinical information, including symptoms and diagnoses..$^{14}$ In addition, THIN also captures basic demographic information, including sex and year of birth. Social deprivation is measured by the Townsend deprivation score (available in quintiles),${ }^{15}$ which is a composite index of owner occupation, car ownership, overcrowding, and unemployment, based on the patient's postcode and information from the 2001 census data. Diagnoses and 
prescriptions are generally well recorded in $\mathrm{THIN}^{16}$ and have been used successfully in previous dementia studies., ${ }^{3,17-19}$

\section{Ethics approval}

Use of THIN for scientific research was approved by the National Health Service (NHS) South-East Multi-Centre Research Ethics Committee in 2003. Scientific approval to undertake this study was obtained from IQVIA World Publications Scientific Review Committee in March 2017 (reference 17THIN019).

\section{Study population}

We included individuals aged 50-105 years contributing data to THIN between January 1, 2007 and December 31, 2015. This was a dynamic cohort, with people entering and exiting the study at different times. Individuals were followed up from the latest of: January 1, 2007; their 50th birthday; 6 months after registration with the general practice (to exclude prevalent cases being newly recorded upon registration with the practice); ${ }^{3}$ or when the practice met standard criteria for acceptable mortality reporting (AMR) and acceptable computer usage (ACU). ${ }^{20,21}$ The end date was defined as the earliest of: the first record indicative of dementia or an antidementia drug prescription; patient's death; patient leaving the practice; practice leaving THIN database; or December 31, 2015.

\section{Main outcome and risk-factor measurements}

To identify incident diagnoses of dementia in THIN, we developed lists of Read codes and antidementia drugs (prescribed only for dementia) and searched medical and prescription records for codes indicative of dementia. We excluded individuals with a diagnosis of dementia before the start of follow-up, as well as those with a diagnosis within the first 6 months after registration, to ensure only incident cases were captured.

Age was analyzed in 10-year age groups (the last age group was 100-105 years, as this was the maximum age included). We examined social deprivation using quintiles of the Townsend deprivation score. ${ }^{15}$ As a proxy measure for comorbidity, we included the prescribing index, which indicates the number of the British National Formulary chapters from which patients received prescriptions (excluding antidementia drugs, vaccines, and anesthetics) and predicts adverse outcomes, as well as more complex comorbidity indices..$^{22}$ We developed code lists to search for ethnicity information in medical and additional health records. Information extracted on ethnicity was grouped into white (British or other white background),
Asian (Indian, Pakistani, Bangladeshi, Chinese, or other Asian background), black (African, Caribbean, or other black background), and mixed/other ethnic groups, based on the Office for National Statistics (ONS) classification. ${ }^{23}$

\section{Statistical analyses}

We estimated the overall crude incidence of dementia diagnosis per 1,000 PYAR by summing the number of patients with a first record indicative of dementia diagnosis between 2007 and 2015 and dividing this by the total number of personyears of follow-up for this period. We also determined crude incidence by calendar year and ethnicity by restricting the number of cases and person-years of follow-up to the respective categories. We estimated incidence rate ratios (IRR) for Asian, black, and mixed/other ethnic groups (compared to the white ethnic group) by fitting multivariable Poisson regression models with log person-time as an offset separately for men and women. We examined relative changes in IRRs for ethnicity after adjusting for age (in 10-year age groups) and calendar year (2007-2015, M0), social deprivation (in quintiles of the Townsend score, M1), the prescribing index (M2), and a binary indicator of diabetes (M3). Models M1M3 incrementally added a given variable to all the variables previously included in M0. All analyses were conducted in Stata version 14.2. ${ }^{24}$ We reported but did not interpret results for the mixed/other ethnic group, as we considered this group to be too heterogeneous to do so.

\section{Multiple imputation of missing data}

Under the assumption of data being missing at random, ${ }^{25} \mathrm{mul}-$ tiple imputation can provide unbiased and statistically more powerful results compared with a complete case analysis by using information from individuals with incomplete data. ${ }^{26}$ However, it was not possible to ascertain whether data were truly missing at random from the observed data alone. To increase the plausibility of the missing at random assumption, we included in our imputation model a wide range of variables that were thought to be predictive of the missing values in ethnicity, as well as the chance of ethnicity being recorded. ${ }^{27}$

We used the multivariate imputation by chained equations algorithm ${ }^{28-30}$ for multiple imputation of missing data in ethnicity and Townsend deprivation score. For each incomplete variable, an imputation model was constructed conditional on variables in the main analysis (indicator of dementia, follow-up time, age group, prescribing index, indicator of diabetes), other disease indicators recorded at any time in the patient records (myocardial infarction, stroke, chronic kidney disease, thalassemia, and schizophrenia), lifestyle factors 
recorded during 1 year before or after the start of follow-up (height, weight, smoking status, alcohol consumption), and the other incomplete variables. Since the lifestyle factors also contained missing values, they were imputed alongside ethnicity and Townsend score in the algorithm. Using appropriate regression models, we examined the association between complete variables (indicator of dementia, follow-up time, age group, prescribing index, indicator of diabetes, and other disease indicators) and incomplete variables (ethnicity, Townsend score, height, weight, smoking status, and alcohol consumption), as well as their binary response indicators, and concluded that data were not likely to be missing completely at random for these incomplete variables. Multiple imputation was performed in Stata version $14.2^{24}$ using the "mi impute chained" 31 command based on the missing at random assumption. After 10 cycles of the chained equations, 20 imputed data sets were created for men and women separately to reflect potential interactions in the main analysis. Each imputed data set was analyzed identically, and results combined using Rubin's rules. . $^{32,33}$

\section{Comparison with community studies}

We compared incidence of dementia diagnosis in black and white people with community incidence of dementia based on bespoke cohort studies and the age-sex structure of the UK population. We did not perform these estimations for people in the Asian ethnic group due to a lack of evidence regarding incidence of dementia in this group. This was performed after missing values in ethnicity had been handled by a complete case analysis or multiple imputation, as described in the previous section.

We used the sex- and age-specific incidences reported by Matthews et $\mathrm{al}^{2}$ from the second phase of the Medical Research Council Cognitive Function \& Ageing Study (CFAS II) as incidences among the white ethnic group. We took the ratios of the incidences of African-American and white non-Latino American reported in a systematic review by Mehta and $\mathrm{Yeo}^{34}$ to estimate the proportions in the black ethnic group (incidence ratio 1.63). This was done based on the assumptions that incidences are relatively similar between US and UK populations; the ethnicity-specific dementia incidence association is broadly similar for females and males across age groups; and THIN is broadly representative of the UK population. The overall UK population by sex and age group in 2015 was obtained from the ONS data, and the UK population by sex and age group for white and black ethnicities was estimated using the UK 2011 census figures for the distribution of ethnicity. ${ }^{35,36}$

For complete case analysis, we first restricted our THIN cohort to individuals with a record of ethnicity. To estimate the number of new dementia diagnoses in THIN, we counted the number of individuals who were actively registered with THIN practices in 2015 by sex, age group, and white and black ethnicities. For the white ethnic group, these counts were then multiplied by the incidence of dementia taken from Matthews et al. ${ }^{2}$ For the black ethnic group, the corresponding counts were multiplied by 1.63 times Matthews et al's estimated incidence of dementia. ${ }^{2}$ We then counted the observed number of individuals who were actively registered with THIN practices in 2015 and received a diagnosis of dementia in 2015 by sex, age group, and white and black ethnicities. This was divided by the corresponding estimated number of dementia diagnoses calculated earlier to obtain the estimated percentage of diagnoses in 2015.

For multiple imputation, after performing multiple imputation of missing values in ethnicity in our cohort, we counted the total number of individuals who were actively registered with THIN practices in 2015 by sex and age group. We then estimated white and black ethnic proportions by sex and age group among these individuals in each imputed data set and combined the results to obtain an overall set of ethnic proportions using Rubin's rules. ${ }^{32,33}$ These proportions were multiplied by the total counts in the previous step to obtain the estimated number of individuals actively registered with THIN practices in 2015 by sex, age group, and white and black ethnicities. To estimate the number of new dementia diagnoses in THIN for the white ethnic group, these were multiplied by the incidence of dementia taken from Matthews et al. ${ }^{2} \mathrm{We}$ followed the same procedure as before to obtain the estimated number of dementia diagnoses in THIN for the black ethnic group. To estimate the number of white and black individuals who were diagnosed with dementia in 2015, we counted the total number of individuals who were diagnosed with dementia in 2015, obtained estimated proportions of white and black individuals with a diagnosis in 2015 from the imputed data sets as before, and multiplied the two together. These numbers were then divided by the estimated number of dementia diagnoses to obtain the estimated percentage of diagnoses in 2015.

To obtain overall percentages of dementia diagnoses for white and black men and women, the estimated percentages obtained from complete case analysis and multiple imputation were age-standardized based on the age distribution by sex and white and black ethnicities in the UK population and our sample.

\section{Results}

We reported the results of our complete case and multipleimputation analyses throughout, but considered the multiple imputation results our primary findings. This was because we identified variables associated with ethnicity and the recording of ethnicity, and made an assumption that missing values 
were missing at random. Under this missingness assumption, multiple imputation analysis can be unbiased and statistically more powerful compared with a complete case analysis.

\section{Complete case analysis}

Of the 2,511,681 older individuals (Table 1), we identified $66,083(3 \%)$ individuals with a record indicating that they had a first diagnosis of dementia between 2007 and 2015. This was equivalent to an incidence of 4.87/1,000 PYAR (95\% CI 4.83-4.90). The overall incidence of dementia increased over time from 3.75 to 5.65 cases per 1,000 PYAR between 2007 and 2015 (Figure 1A). Among those diagnosed with dementia, 36,439 (55\%) individuals had missing ethnicity; for those whom ethnicity was known, 28,672 (97\%) were in the white ethnic group, 480 (2\%) in the Asian ethnic group, 315 $(1 \%)$ in the black ethnic group, and $177(<1 \%)$ in the mixed/ other group. Overall, for individuals with a record of ethnicity $(1,178,111,47 \%)$, the annual incidence of dementia diagnosis also followed an upward trend and appeared comparable between the white, Asian, and black ethnic groups (Figure 1B). Townsend deprivation score was available for $2,452,173$ (98\%) individuals. Restricting the analysis to the complete cases (those without missing values in ethnicity and Townsend score) reduced the sample size to $1,144,195$ individuals with 28,795 cases of dementia, corresponding to an incidence of 4.64/1,000 PYAR (95\% CI 4.59-4.70).

Table I Summary of variables in the main analysis

\begin{tabular}{|c|c|}
\hline Variables & Summary statistics \\
\hline \multicolumn{2}{|l|}{ Sex } \\
\hline Men & I, I97,948 (48) \\
\hline Women & $\mathrm{I}, 313,733(52)$ \\
\hline Age at start, median (IQR), years & $59.5(51.5-70.5)$ \\
\hline \multicolumn{2}{|l|}{ Townsend deprivation score } \\
\hline Quintile I (least deprived) & $665,484(27)$ \\
\hline Quintile 2 & $587,249(23)$ \\
\hline Quintile 3 & $515,931(20)$ \\
\hline Quintile 4 & $4 \mid 5,825(17)$ \\
\hline Quintile 5 (most deprived) & $267,684(\mathrm{II})$ \\
\hline Missing & $59,508(2)$ \\
\hline Diabetes & $825,926(33)$ \\
\hline Prescribing index, median (IQR) & $8(5-10)$ \\
\hline \multicolumn{2}{|l|}{ Ethnicity } \\
\hline White & $\mathrm{I}, \mathrm{I} \mid \mathrm{I}, 840(44)$ \\
\hline Asian & $31,757(I)$ \\
\hline Black & $18,214(1)$ \\
\hline Mixed/other & $15,300(1)$ \\
\hline Missing & $\mathrm{I}, 333,570(53)$ \\
\hline Dementia & $66,083(3)$ \\
\hline Follow-up, median (IQR), years & $5.5(2.6-8.6)$ \\
\hline Total & $2,511,681(100)$ \\
\hline
\end{tabular}

Notes: Statistics displayed as frequency (percentage) or median (IQR). There were $2,441,768(97 \%)$ individuals with at least one prescription in their electronic health records (prescribing index $>0$ ).

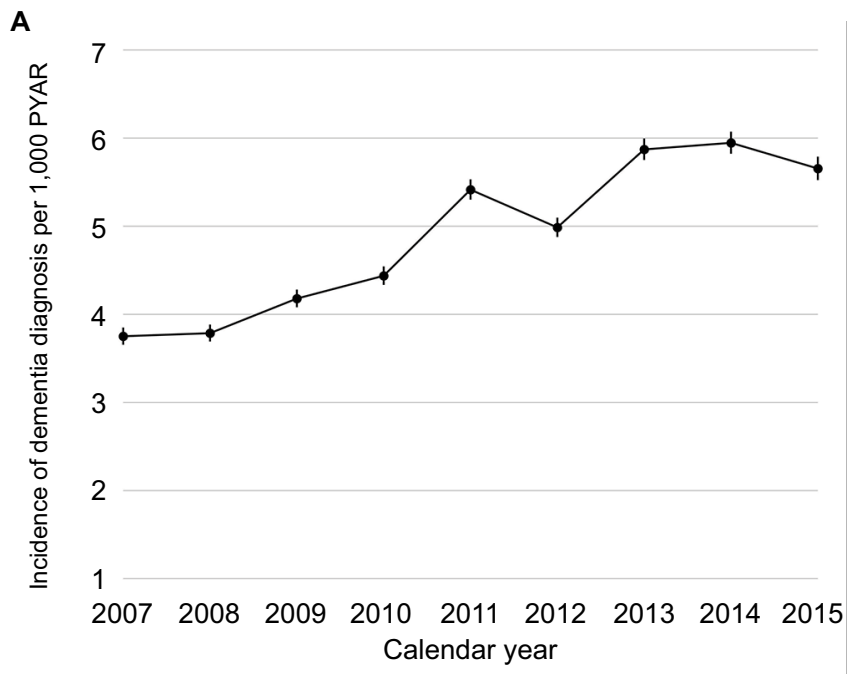

B

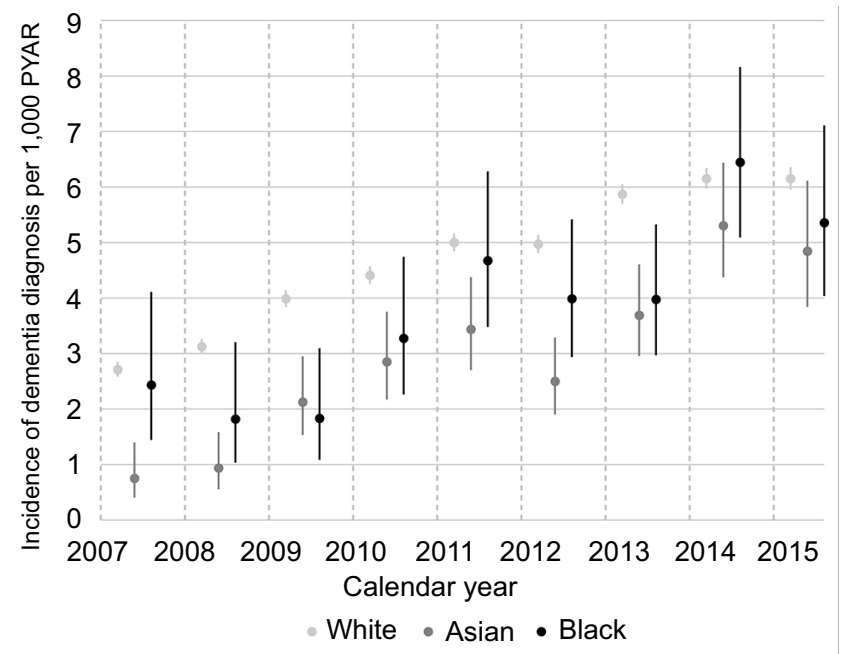

Figure I Incidence of dementia diagnosis per I,000 PYAR by calendar year in the Health Improvement Network UK primary care database.

Notes: (A) Incidence among overall cohort $(n=2,5 \mathrm{I}, \mathrm{68}$ I); (B) incidence for white, Asian, and black ethnic groups among individuals with at least one record of ethnicity $(\mathrm{n}=\mathrm{I}, \mathrm{I78}, \mathrm{III})$. Error bars represent $95 \% \mathrm{Cls}$.

Abbreviation: PYAR, person-years at risk.

Table 2 show adjusted IRRs of dementia for Asian, black, and mixed/other ethnic groups (compared with the white ethnic group) from multivariable Poisson regression models, and results are presented separately for men and women. In the first model adjusted for age and calendar year (M0), incidence of dementia diagnosis appeared to be similar between white and Asian ethnic groups (IRR 1.01, 95\% CI 0.88-1.15 for men; IRR 0.96, 95\% CI 0.84-1.09 for women). Incidence of dementia diagnosis among black men was 1.31 times higher than that among white men $(95 \% \mathrm{CI}$ 1.10-1.56), and similarly a higher incidence was seen among black women compared with white women (IRR 1.38, 95\% CI 1.19-1.60). Adjusting for the Townsend deprivation score (M1), prescribing index (M2), and indicator of diabetes (M3) 
Table 2 Complete case analysis

\begin{tabular}{|c|c|c|c|c|c|}
\hline \multirow[t]{2}{*}{ Model } & \multirow[t]{2}{*}{ Ethnicity } & \multicolumn{2}{|c|}{$\begin{array}{l}\text { Men } \\
(n=536,920)\end{array}$} & \multicolumn{2}{|c|}{$\begin{array}{l}\text { Women } \\
(n=607,275)\end{array}$} \\
\hline & & IRR & $95 \% \mathrm{Cl}$ & IRR & $95 \% \mathrm{Cl}$ \\
\hline \multirow[t]{4}{*}{ Mo } & White & 1 & & I & \\
\hline & Asian & 1.01 & $0.88-1.15$ & 0.96 & $0.84-1.09$ \\
\hline & Black & 1.31 & $1.10-1.56$ & 1.38 & $1.19-1.60$ \\
\hline & Mixed/other & 0.87 & $0.69-1.11$ & 0.91 & $0.75-1.11$ \\
\hline \multirow[t]{4}{*}{ MI } & White & 1 & & 1 & \\
\hline & Asian & 0.96 & $0.84-1.10$ & 0.92 & $0.81-1.05$ \\
\hline & Black & 1.20 & $1.01-1.43$ & 1.29 & $1.11-1.49$ \\
\hline & Mixed/other & 0.85 & $0.67-1.07$ & 0.90 & $0.73-1.10$ \\
\hline \multirow[t]{4}{*}{$\overline{M 2}$} & White & 1 & & I & \\
\hline & Asian & 0.90 & $0.79-1.03$ & 0.91 & $0.80-1.04$ \\
\hline & Black & 1.20 & I.00-1.42 & 1.30 & $|| 2-1.5 \mid$. \\
\hline & Mixed/other & 0.86 & $0.68-1.09$ & 0.91 & $0.75-1.11$ \\
\hline \multirow[t]{4}{*}{ M3 } & White & $\mathrm{I}$ & & I & \\
\hline & Asian & 0.87 & $0.76-0.99$ & 0.88 & $0.77-0.99$ \\
\hline & Black & 1.15 & $0.97-1.37$ & 1.24 & I.07-I.44 \\
\hline & Mixed/other & 0.85 & $0.67-1.08$ & 0.90 & $0.74-1.10$ \\
\hline
\end{tabular}

Notes: IRRs of dementia diagnosis for Asian, black, and mixed/other ethnic groups (compared with the white ethnic group) from multivariable Poisson regression models adjusted for age (in 10-year age groups) and calendar year (2007-2015, M0); Townsend deprivation score (in quintiles, MI); prescribing index (M2); and binary indicator of diabetes (M3), stratified by sex; $n=1,144,195$. Models MI-M3 incrementally added a given variable to all the variables previously included in MO. Abbreviation: IRR, incidence rate ratio.

reduced the estimated IRRs for the Asian and black ethnic groups in both men and women.

In the fully adjusted model (M3), incidence was found to be slightly lower for the Asian ethnic group compared with the white ethnic group in both men and women (IRR 0.87, 95\% CI 0.76-0.99 for men; IRR 0.88, 95\% CI 0.77-0.99 for women). The black ethnic group was associated with a higher incidence of dementia diagnosis compared with the white ethnic group, and this association was stronger for women compared with men (IRR $1.15,95 \%$ CI $0.97-1.37$ for men; IRR 1.24, 95\% CI 1.07-1.44 for women).

\section{Multiple imputation analysis}

There were 471,433 (19\%) individuals who had complete data in ethnicity, Townsend deprivation score, height, weight, smoking status, and alcohol consumption. In total, 1,333,570 (53\%) missing values in ethnicity, 59,508 (2\%) missing values in Townsend deprivation score, 241,614 (10\%) missing values in height, 1,142,332 (46\%) missing values in weight, 702,647 (28\%) missing values in smoking status, and 1,565,757 (62\%) missing values in alcohol consumption were imputed.

Results of the multiple imputation analysis were comparable with that of the complete case analysis and are presented in Table 3 for men and women separately, including the ethnicity-specific IRRs of dementia diagnosis adjusted for age group, calendar year, Townsend deprivation score, prescribing index, and indicator of diabetes (M3). After multiple imputation, incidence of dementia diagnosis was found to be lower among Asian people compared with their white counterparts, particularly for women (IRR $0.88,95 \% \mathrm{CI}$ 0.76-1.01 for men; IRR $0.82,95 \%$ CI $0.72-0.95$ for women). In contrast, the black ethnic group had higher incidence compared with the white ethnic group, and the association was slightly stronger for men (IRR $1.28,95 \%$ CI $1.08-1.50$ for men; IRR $1.25,95 \%$ CI 1.07-1.46 for women).

\section{Comparison with community studies}

Table 4 presents the projected age-standardized percentages of dementia diagnoses among individuals who were actively

Table 3 Multiple imputation analysis

\begin{tabular}{|c|c|c|c|c|}
\hline \multirow[t]{2}{*}{ Variables } & \multicolumn{2}{|c|}{$\begin{array}{l}\text { Men } \\
(n=I, 197,948)\end{array}$} & \multicolumn{2}{|c|}{$\begin{array}{l}\text { Women } \\
(n=1,3 \mid 3,733)\end{array}$} \\
\hline & IRR & $95 \% \mathrm{Cl}$ & IRR & $95 \% \mathrm{Cl}$ \\
\hline \multicolumn{5}{|l|}{ Ethnicity } \\
\hline White & I & & I & \\
\hline Asian & 0.88 & $0.76-1.01$ & 0.82 & $0.72-0.95$ \\
\hline Black & 1.28 & I.08-1.50 & 1.25 & I.07-1.46 \\
\hline Mixed/other & 0.86 & $0.69-1.08$ & 0.97 & $0.80-1.18$ \\
\hline \multicolumn{5}{|l|}{ Age group, years } \\
\hline $50-59$ & 0.05 & $0.05-0.06$ & 0.04 & $0.04-0.05$ \\
\hline $60-69$ & 0.23 & $0.22-0.24$ & 0.19 & $0.18-0.19$ \\
\hline 70-79 & I & & 1 & \\
\hline $80-89$ & 2.81 & $2.73-2.89$ & 3.23 & $3.16-3.31$ \\
\hline $90-99$ & 4.02 & $3.83-4.22$ & 4.77 & $4.63-4.92$ \\
\hline $100+$ & 3.30 & $2.19-4.97$ & 3.25 & $2.74-3.87$ \\
\hline \multicolumn{5}{|l|}{ Year } \\
\hline 2007 & I & & I & \\
\hline 2008 & 1.04 & II-I. & 0.99 & $0.95-1.04$ \\
\hline 2009 & 1.21 & $1.14-1.29$ & 1.07 & $1.02-1.12$ \\
\hline 2010 & 1.26 & I.18-1.33 & 1.15 & $1.10-1.20$ \\
\hline 2011 & 1.59 & $1.51-1.69$ & 1.38 & I.32-1.44 \\
\hline 2012 & 1.45 & I.37-I.54 & 1.29 & $1.23-1.34$ \\
\hline 2013 & 1.82 & $1.72-1.92$ & 1.48 & $1.42-1.54$ \\
\hline 2014 & 1.83 & $1.73-1.93$ & 1.52 & $1.45-1.58$ \\
\hline 2015 & 1.74 & I.64-I.84 & 1.48 & $1.42-1.55$ \\
\hline \multicolumn{5}{|l|}{ Townsend deprivation } \\
\hline Quintile I (least deprived) & I & & I & \\
\hline Quintile 2 & 1.10 & $1.06-1.14$ & 1.06 & $1.03-1.09$ \\
\hline Quintile 3 & 1.19 & $1.15-1.23$ & 1.13 & $1.10-1.17$ \\
\hline Quintile 4 & 1.25 & $1.20-1.30$ & 1.19 & $1.16-1.23$ \\
\hline Quintile 5 (most deprived) & 1.30 & $1.24-1.36$ & 1.23 & $1.19-1.28$ \\
\hline Prescribing index & 1.08 & $1.07-1.08$ & 1.03 & $1.03-1.03$ \\
\hline \multicolumn{5}{|l|}{ Diabetes } \\
\hline No & I & & I & \\
\hline Yes & 1.15 & $1.12-1.18$ & 1.16 & $1.14-1.18$ \\
\hline
\end{tabular}

Notes: IRRs of dementia diagnosis for Asian, black, and other ethnic groups (compared with the white ethnic group) from a multivariable Poisson regression model adjusted for 10-year age groups, calendar year (2007-2015), Townsend deprivation score (in quintiles), prescribing index, and binary indicator for diabetes, stratified by sex; $n=2,511,681$.

Abbreviation: IRR, incidence rate ratio. 


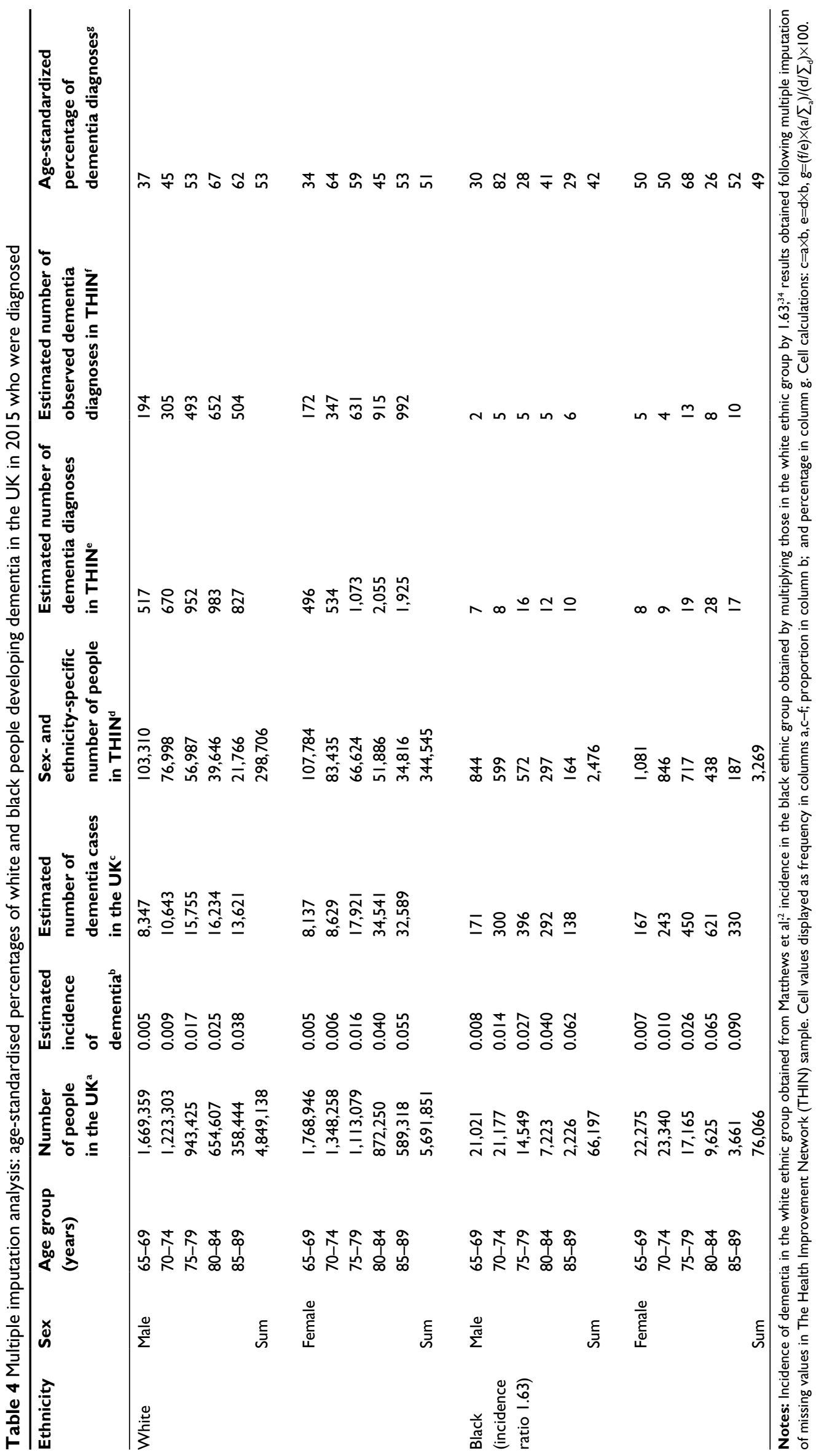


registered in 2015, after multiple imputation of missing values. The percentages of dementia diagnoses were $49 \%$ and $51 \%$ for women of black and white ethnicities, respectively. For black and white men, these were estimated to be $42 \%$ and $53 \%$, respectively. Percentages of dementia diagnoses in the complete case analysis were comparable but slightly lower, since individuals diagnosed with dementia who did not have a record of ethnicity were not included in the calculations (Table S1).

\section{Discussion}

To the best of our knowledge, this is the first study to compare incidence of dementia diagnosis among ethnic groups in a nationally representative sample and to evaluate whether ethnicity is associated with the likelihood of people with dementia receiving a diagnosis. We found that compared with the white ethnic group, Asian women were 18\% less likely and Asian men $12 \%$ less likely to have a new dementia diagnosis. By contrast, black women were $25 \%$ more likely and black men $28 \%$ more likely to receive a new dementia diagnosis compared with the white ethnic group. Through comparison with community cohort studies, we estimated that the increased incidence of dementia in black men was only partly reflected in these diagnoses, and that black men with dementia were less likely to receive a diagnosis compared with white men. In black and white women with dementia, we estimated that the likelihood of receiving a diagnosis of dementia was broadly comparable.

The only community cohort studies in Asian populations have been in Japanese-American people. ${ }^{7}$ These indicated that dementia incidence is lower than or comparable with the white majority ethnic groups. It is thus possible that the lower incidence of dementia diagnosis we found in Asian people after controlling for morbidity and area deprivation reflected lower dementia incidence. Several known lifestyle and environmental risk factors for dementia are more common in Asian compared with white majority groups in the UK. ${ }^{7}$ These include lower levels of formal education, which we were not able to measure, as well as cardiovascular risk factors. Differing levels of genetic susceptibility among ethnic groups are one possible explanation for the apparent paradox of lower dementia diagnosis incidence in a population with more prevalent environmental and lifestyle risk factors. ${ }^{7}$ Lower incidence of dementia diagnosis in Asian people is another explanation.

Our finding that black men with dementia appeared relatively less likely to receive a diagnosis of dementia than men from the white majority population is concerning. A previous qualitative study of attitudes to dementia reported a perception among people from black ethnic groups in the UK that dementia was "a white person's illness", that seeking medical help was pointless or only for severe problems, and that dementia was a private and stigmatizing problem. ${ }^{37}$ Our results could indicate that black men experience barriers to accessing health services or receiving a diagnosis. Family concerns about previous negative experiences of psychiatric services may be particularly significant in BME groups. Clinicians may be more reluctant to diagnose dementia in BME groups, due to awareness of cultural bias in standard cognitive tests, especially if appropriate culturally competent tests are unavailable. ${ }^{38}$ In UK South Asian people, it has been suggested that delayed help-seeking for dementia might be explained by stigma linked with ideas of "madness", lack of physical etiology and lack of treatment, as well as fear of moves to long-term care. ${ }^{39}$

Our findings suggested a small rise in age-adjusted incidence of dementia diagnosis since 2011. The most recent population-based studies have reported stable or declining age-standardized dementia prevalence over time. The rise in likelihood of people with dementia receiving a diagnosis over this time probably explains our findings. The proportion of people with dementia who were diagnosed has risen significantly over this time, in response to a major national program to improve diagnosis rates: from an estimated $42 \%$ in $2010-2011$ to $67 \%$ by $2015 .^{40}$

Strengths of this study included the large, representative sample: THIN database comprises around $6 \%$ of the UK population and has been found to be broadly representative in terms of demographic and health variables, although slightly more THIN patients live in affluent areas compared with the general population. ${ }^{13}$ We reported findings from our multiple imputation analysis as our primary results, because they were inclusive of a greater proportion of the cohort (sample size was increased from 1,144,195 in a complete case analysis to $2,511,681$ in the multiple imputation analysis). We also reported findings from a complete case analysis that were comparable with those in multiple imputation. Complete case analysis generally showed smaller differences in incidence of dementia diagnosis among ethnic groups. Most noticeably, the difference in incidence of dementia diagnosis between black and white men was smaller among the complete cases compared with the multiple imputation analysis.

Our primary exposure was self-reported ethnic group. We reported ethnicity as black, Asian, white, or mixed/other, as data at more detailed levels were less well recorded. Ethnicity is a complex construct that varies in definition over time. 
Ethnic groups are considered those sharing a common ancestry, culture, historical memories, attachment to a homeland, and feeling of solidarity with one another. There is wide variation within minority ethnic groups in country of origin, language, religion, socioeconomic power, and experiences, but there is also enough shared culture with regard to family structures, identity, and health beliefs to make ethnicity a relevant factor with respect to health behaviors. ${ }^{41}$ Although we attempted to account for the uncertainty associated with incomplete information on ethnicity by using an increased number of imputations (20 imputed data sets) and including many variables in the imputation model for ethnicity to enhance the plausibility of the missing at random assumption, results still need to be interpreted with caution, due the relatively high level of missing data on ethnicity.

Our estimates of the proportion of people with dementia receiving diagnoses assumed that the ratios of incidence of dementia between UK black and white ethnic groups were comparable with those reported between African-American and white non-Latino American people in a large metaanalysis. ${ }^{34}$ This was a conservative estimate of the ratio: it excluded Hispanic-Caribbean populations, who have a higher incidence of dementia than African-American groups. ${ }^{34}$ Over two-thirds of African-Americans in the USA are of West African heritage ${ }^{42}$ whereas in the UK 2011 census, just over half the people who identified as being from a black ethnic group identified as black African, with black Caribbean the second-most common group. It is possible that if dementia risk is similarly increased in UK black Caribbean relative to black African groups, our incidence estimates for the black ethnicity may have been an underestimate. Our assumption that ethnicity-specific dementia-incidence association is broadly similar for females and males across age groups is based on the absence of evidence of any sex effect; however, lack of evidence of effect is not evidence of lack of effect.

The estimated proportions of dementia cases that were diagnosed in general practitioner (GP) records among black and white ethnic groups were lower than have been ascertained from other methods. Although it was estimated from national data that $67 \%$ of people living with dementia in 2015 received a diagnosis, ${ }^{40}$ we estimated that only around half the people with dementia received a diagnosis. Our estimates were based on an assumption that THIN sample was nationally representative, but there is evidence that people living in more deprived areas may be underrepresented in THIN. ${ }^{13}$ As dementia is more common in more deprived areas, ${ }^{18}$ this could explain why our diagnosis incidence estimates were lower than those reported in national data. As BME people may be more likely to live in deprived areas, incidence of dementia diagnosis reported in BME groups may have been an underestimate. There is also some evidence that dementia is underrecorded in GP records across ethnic groups. ${ }^{3}$ The use of an area-level deprivation measure is a limitation, as individual deprivation level can sometimes be at odds with the deprivation level of the area in which a person lives.

In our previous dementia studies using THIN data, ${ }^{18,19}$ we found that accounting for practice-level variation had little effect on the results. Because of this, and the complexities of performing a multiple imputation analysis that reflects the clustering structure of the analysis model, we did not examine variation across general practices in this paper. We could not identify people with undiagnosed dementia or control for dementia severity. Although we reported underdiagnosis in nonwhite ethnic groups, there is some evidence that false-positive dementia diagnoses may be more common in UK minority ethnic groups because standard cognitive assessments underestimate abilities. ${ }^{43}$ Although diagnoses of dementia are often made in memory services in the UK, there may be variation between primary care practices in how dementia diagnoses are made and recorded in BME groups, eg, in interpreter availability.

\section{Conclusion}

In this first study to report dementia diagnosis rates among ethnic groups in a large nationally representative sample, we found that people from Asian UK ethnic groups were less likely and those from black ethnic groups more likely to receive a dementia diagnosis compared with the white majority population. By comparing these incidence estimates to those from community cohort studies, we concluded that the increased incidence of dementia diagnosis in black men only partly reflects their higher dementia incidence. We estimated that black men with dementia were around $10 \%$ less likely to be diagnosed than white men. The lower incidence of dementia diagnosis in Asian populations may reflect underdiagnosis or lower incidence of dementia. A community cohort study is needed to determine how dementia incidence may differ among ethnic groups, especially between Asian and white majority ethnic groups.

\section{Acknowledgments}

This work was supported by The Dunhill Medical Trust (grant number R530/1116). The funders were not involved in study design, collection, analysis, interpretation of data, writing of the article, and the decision to submit it for publication; the researchers were independent from the funders and sponsors 
in carrying out this work. Abstract was published on British Society of Gerontology 47th Annual Conference - Ageing in an Unequal World: Shaping Environments for the 21st Century. (https://app.oxfordabstracts.com/stages/25/programme-builder/ submission/16145?backHref=/events/28/sessions/84).

\section{Author contributions}

All authors contributed toward data analysis, drafting and revising the paper and agree to be accountable for all aspects of the work.

\section{Disclosure}

CC and NM are supported by the University College London Hospitals NHS Foundation Trust (UCLH) National Institute for Health Research (NIHR) Biomedical Research Centre. RR was (in part) supported by the NIHR Collaboration for Leadership in Applied Health Research and Care (CLAHRC) North Thames at Bart's Health NHS Trust. The views expressed are those of the author(s) and not necessarily those of the NHS, the NIHR or the Department of Health and Social Care. The authors report no other conflicts of interest in this work.

\section{References}

1. Prince M, Comas-Herrera A, Knapp M, Guerchet M, Karagiannidou M. World Alzheimer Report 2016: Improving Healthcare for People Living with Dementia. London: Alzheimer's Disease International; 2016.

2. Matthews FE, Stephan BC, Robinson L, et al. A two decade dementia incidence comparison from the Cognitive Function and Ageing Studies I and II. Nat Commun. 2016;7:11398.

3. Rait G, Walters K, Bottomley C, Petersen I, Iliffe S, Nazareth I. Survival of people with clinical diagnosis of dementia in primary care: cohort study. BMJ. 2010;341:c3584.

4. Cooper C, Tandy AR, Balamurali TB, Livingston G. A systematic review and meta-analysis of ethnic differences in use of dementia treatment care and research. Am J Geriatr Psychiatry. 2010;18:193-203.

5. Prince M, Knapp M, Guerchet M, et al. Dementia UK: Second Edition - Overview. London: Alzheimer's Society; 2014.

6. Gordon-Larsen P, Popkin B. Understanding socioeconomic and racial/ ethnic status disparities in diet exercise and weight: underlying contextual factors and pathways. J Am Diet Assoc. 2011;111:1816-1819.

7. Mayeda ER, Glymour MM, Quesenberry CP, Whitmer RA. Heterogeneity in 14-year dementia incidence between Asian American subgroups. Alzheimer Dis Assoc Disord. 2017;31:181-186.

8. All-Party Parliamentary Group on Dementia. Dementia Does Not Discriminate: The Experiences of Black Asian and Minority Ethnic Communities. London: All-Party Parliamentary Group on Dementia; 2013.

9. Adelman S, Blanchard M, Rait G, Leavey G, Livingston G. Prevalence of dementia in African-Caribbean compared with UK-born white older people: two-stage cross-sectional study. Br J Psychiatry. 2011;199:119-125.

10. Boreinstein AR, Wu Y, Bowen JD, et al. Incidence rates of dementia, Alzheimer disease, and vascular dementia in the Japanese American population in Seattle, WA: the Kame project. Alzheimer Dis Assoc Disord. 2014;28:23-29.

11. Mayeda ER, Glymour MM, Quesenberry CP, Whitmer RA. Inequalities in dementia incidence between six racial and ethnic groups over 14 years. Alzheimers Dement. 2016;12:216-224.
12. IMS Health. Our data. 2015. Available at: https://www.iqvia.com/. Accessed 22 April 2018.

13. Blak BT, Thompson M, Dattani H, Bourke A. Generalisability of the Health Improvement Network (THIN) database: demographics chronic disease prevalence and mortality rates. Inform Prim Care. 2011;19:251-255.

14. Chisholm J. The Read clinical classification. BMJ. 1990;300:1092.

15. Townsend P, Phillimore P, Beattie A. Health and Deprivation: Inequality and the North. London: Croom Helm; 1987.

16. Lewis JD, Schinnar R, Bilker WB, Wang X, Strom BL. Validation studies of the Health Improvement Network (THIN) database for pharmacoepidemiology research. Pharmacoepidemiol Drug Saf. 2007;16:393-401.

17. Walters K, Hardoon S, Petersen I, et al. Predicting dementia risk in primary care: development and validation of the dementia risk score using routinely collected data. BMC Med. 2016;14:6.

18. Cooper C, Lodwick R, Walters K, et al. Observational cohort study: deprivation and access to anti-dementia drugs in the UK. Age Ageing. 2016;45:148-154.

19. Cooper $\mathrm{C}$, Lodwick $\mathrm{R}$, Walters $\mathrm{K}$, et al. Inequalities in receipt of mental and physical healthcare in people with dementia in the UK. Age Ageing. 2017;46:393-400.

20. Maguire A, Blak BT, Thompson M. The importance of defining periods of complete mortality reporting for research using automated data from primary care. Pharmacoepidemiol Drug Saf. 2009;18:76-83.

21. Horsfall L, Walters K, Petersen I. Identifying periods of acceptable computer usage in primary care research databases. Pharmacoepidemiol Drug Saf. 2013;22:64-69.

22. Brilleman SL, Salisbury C. Comparing measures of multimorbidity to predict outcomes in primary care: a cross sectional study. Fam Pract. 2013;30:172-178.

23. Office for National Statistics. Ethnicity and national identity in England and Wales: 2011. 2012. Available from: https://www.ons.gov.uk/ peoplepopulationandcommunity/culturalidentity/ethnicity/articles/ ethnicityandnationalidentityinenglandandwales/2012-12-11. Accessed May 30, 2018.

24. StataCorp. Stata 14 [software]. 2015. Available from: https://www.stata. com/stata14. Accessed May 30, 2018.

25. Rubin DB. Inference and missing data. Biometrika. 1976;63:581-592.

26. Sterne JA, White IR, Carlin JB, et al. Multiple imputation for missing data in epidemiological and clinical research: potential and pitfalls. BMJ. 2009;338:b2393.

27. Collins LM, Schafer JL, Kam CM. A comparison of inclusive and restrictive strategies in modern missing data procedures. Psychol Methods. 2001;6:330-351.

28. van Buuren S, Boshuizen HC, Knook DL. Multiple imputation of missing blood pressure covariates in survival analysis. Stat Med. 1999;18:681-694.

29. van Buuren S, Brand JP, Groothuis-Oudshoorn CG, Rubin DB. Fully conditional specification in multivariate imputation. J Stat Comput Simul. 2006;76:1049-1064.

30. White IR, Royston P, Wood AM. Multiple imputation using chained equations: issues and guidance for practice. Stat Med. 2011;30:377-399.

31. StataCorp. Stata multiple-imputation reference manual: release 14. 2015. Available from: https://www.stata-press.com/data/r14/mimain. html. Accessed May 30, 2018.

32. Rubin DB. Multiple Imputation for Nonresponse in Surveys. Hoboken, NJ: Wiley; 1987.

33. Barnard J, Rubin DB. Small-sample degrees of freedom with multiple imputation. Biometrika. 1999;86:948-955.

34. Mehta KM, Yeo GW. Systematic review of dementia prevalence and incidence in United States race/ethnic populations. Alzheimers Dement. 2017;13:72-83.

35. Office for National Statistics. Population estimates for UK, England and Wales, Scotland and Northern Ireland. 2015. Available from: https:// www.ons.gov.uk/peoplepopulationandcommunity/populationandmigration/populationestimates/datasets/populationestimatesforukenglandandwalesscotlandandnorthernireland. Accessed May 30, 2018. 
36. Office for National Statistics. Ethnic group. 2015. Available from: http://www.ons.gov.uk/ons/guide-method/harmonisation/primary-setof-harmonised-concepts-and-questions/ethnic-group.pdf. Accessed May 30, 2018.

37. Berwald S, Roche M, Adelman S, Mukadam N, Livingston G. Black African and Caribbean British communities' perceptions of memory problems: 'We don't do dementia'. PLoS One. 2016;11:e0151878.

38. Cooper C, Regan C, Tandy AR, Johnson S, Livingston G. Acute mental health care for older people by crisis resolution teams in England. Int J Geriatr Psychiatry. 2007;22:263-265.

39. Mukadam N, Waugh A, Cooper C, Livingston G. What would encourage help-seeking for memory problems among UK-based South Asians? A qualitative study. BMJ Open. 2015;5:e007990.
40. Parkin E, Baker C. Dementia: policy services and statistics. 2016. Available from: researchbriefings.files.parliament.uk/documents/SN07007/ SN07007.pdf. Accessed May 30, 2018.

41. Mukadam N, Cooper C, Basit B, Livingston G. Why do ethnic elders present later to UK dementia services? A qualitative study. Int Psychogeriatr. 2011;23:1070-1077.

42. Bryc K, Durand EY, Macpherson JM, Reich D, Mountain JL. The genetic ancestry of African Americans, Latinos, and European Americans across the United States. Am J Hum Genet. 2015;96:37-53.

43. Khan F, Tadros G. Complexity in cognitive assessment of elderly British minority ethnic groups: cultural perspective. Dementia. 2014; $13: 467-482$ 


\section{Supplementary material}

Table SI Complete case analysis: age-standardized percentages of white and black people developing dementia in the UK in 2015 who were diagnosed

\begin{tabular}{|c|c|c|c|c|c|c|c|c|c|}
\hline Ethnicity & Sex & $\begin{array}{l}\text { Age group } \\
\text { (years) }\end{array}$ & $\begin{array}{l}\text { Number } \\
\text { of people } \\
\text { in the } \\
\text { UK }\end{array}$ & $\begin{array}{l}\text { Estimated } \\
\text { incidence of } \\
\text { dementia }^{b}\end{array}$ & $\begin{array}{l}\text { Estimated } \\
\text { number of } \\
\text { dementia } \\
\text { cases in the } \\
\text { UKc }\end{array}$ & $\begin{array}{l}\text { Sex- and } \\
\text { ethnicity- } \\
\text { specific } \\
\text { number of } \\
\text { people in } \\
\text { THIN }^{d}\end{array}$ & $\begin{array}{l}\text { Estimated } \\
\text { number of } \\
\text { dementia } \\
\text { diagnoses } \\
\text { in THIN }\end{array}$ & $\begin{array}{l}\text { Number } \\
\text { of } \\
\text { observed } \\
\text { dementia } \\
\text { diagnoses } \\
\text { in THIN }\end{array}$ & $\begin{array}{l}\text { Age- } \\
\text { standardized } \\
\text { percentage } \\
\text { of dementia } \\
\text { diagnoses }^{g}\end{array}$ \\
\hline \multirow[t]{12}{*}{ White } & Male & $65-69$ & $1,669,359$ & 0.005 & 8,347 & 83,705 & 419 & 113 & 30 \\
\hline & & $70-74$ & $1,223,303$ & 0.009 & 10,643 & $65,67 \mid$ & 571 & 178 & 32 \\
\hline & & $75-79$ & 943,425 & 0.017 & 15,755 & 50,698 & 847 & 261 & 31 \\
\hline & & $80-84$ & 654,607 & 0.025 & 16,234 & 38,446 & 953 & 352 & 34 \\
\hline & & $85-89$ & 358,444 & 0.038 & $|3,62|$ & 27,165 & 1,032 & 255 & 18 \\
\hline & Sum & & $4,849,138$ & & & 265,685 & & & 29 \\
\hline & Female & $65-69$ & I,768,946 & 0.005 & 8,137 & 86,934 & 400 & 115 & 31 \\
\hline & & $70-74$ & $1,348,258$ & 0.006 & 8,629 & 68,781 & 440 & 185 & 43 \\
\hline & & $75-79$ & I,II3,079 & 0.016 & $|7,92|$ & 56,380 & 908 & 334 & 38 \\
\hline & & $80-84$ & 872,250 & 0.040 & 34,541 & 46,983 & $|, 86|$ & 481 & 25 \\
\hline & & $85-89$ & 589,318 & 0.055 & 32,589 & 39,893 & 2,206 & 495 & 17 \\
\hline & Sum & & $5,691,851$ & & & 298,971 & & & 31 \\
\hline Black & Male & $65-69$ & 21,021 & 0.008 & 171 & 642 & 5 & I & 25 \\
\hline (incidence & & $70-74$ & 21,177 & 0.014 & 299 & 574 & 8 & 4 & 73 \\
\hline \multirow[t]{10}{*}{ ratio I.63) } & & $75-79$ & 14,549 & 0.027 & 395 & 735 & 20 & 2 & 8 \\
\hline & & $80-84$ & 7,223 & 0.040 & 291 & 435 & 18 & 3 & 11 \\
\hline & & $85-89$ & 2,226 & 0.062 & 137 & 281 & 17 & 4 & 7 \\
\hline & Sum & & 66,197 & & & 2,667 & & & 25 \\
\hline & Female & $65-69$ & 22,275 & 0.007 & 167 & 1,004 & 8 & 4 & 57 \\
\hline & & $70-74$ & 23,340 & 0.010 & 243 & 935 & 10 & 2 & 25 \\
\hline & & $75-79$ & 17,165 & 0.026 & 449 & 894 & 23 & 9 & 36 \\
\hline & & $80-84$ & 9,625 & 0.064 & 619 & 566 & 36 & 4 & 9 \\
\hline & & $85-89$ & 3,661 & 0.090 & 329 & 261 & 23 & 7 & 20 \\
\hline & Sum & & 76,066 & & & 3,660 & & & 29 \\
\hline
\end{tabular}

Notes: Incidence in the white ethnic group obtained from Matthews et al;' incidence in black ethnic group obtained by multiplying that in the white ethnic group by I.63; results obtained following complete case analysis excluding individuals with missing data in The Health Improvement Network (THIN) sample. Cell values displayed as frequency in columns a,c-f; proportion in column b; and percentage in column g. Cell calculations: c=a $\times b, e=d \times b, g=(f / e) \times\left(a / \sum_{a}\right) /\left(d / \sum_{d}\right) \times 100$.

\section{References}

1. Matthews FE, Stephan BC, Robinson L, et al. A two decade dementia incidence comparison from the Cognitive Function and Ageing Studies I and II. Nat Commun. 2016;7:11398.
2. Mehta KM, Yeo GW. Systematic review of dementia prevalence and incidence in United States race/ethnic populations. Alzheimers Dement. 2017;13:72-83.
Clinical Epidemiology

\section{Publish your work in this journal}

Clinical Epidemiology is an international, peer-reviewed, open access, online journal focusing on disease and drug epidemiology, identification of risk factors and screening procedures to develop optimal preventative initiatives and programs. Specific topics include: diagnosis, prognosis, treatment, screening, prevention, risk factor modification,

\section{Dovepress}

systematic reviews, risk and safety of medical interventions, epidemiology and biostatistical methods, and evaluation of guidelines, translational medicine, health policies and economic evaluations. The manuscript management system is completely online and includes a very quick and fair peer-review system, which is all easy to use. 